\title{
A DISCRIMINANT CRITERION FOR AN EQUIVALENCE OF AN ANALYTIC FAMILY OF PLANE CURVE SINGULARITIES AND ITS APPLICATIONS*
}

\author{
CHUNGHYUK KANG ${ }^{\dagger}$ \\ Dedicated to Prof. Henry B. Laufer's 60-th birthday
}

\begin{abstract}
In this paper, we generalize Zariski's discriminant criterion under which an analytic family of plane curve singularities are equivalent. Furthermore, we give a necessary condition of local irreducibility of plane curves with singularities.
\end{abstract}

Key words. topological equivalence of plane curve singularities, the standard Puiseux expansion, the Milnor number.

AMS subject classifications. Primary 32S15, 14E15.

1. Introduction. Throughout this paper, let ${ }_{n} \mathcal{O}$ or $\mathbb{C}\left\{z_{1}, \ldots, z_{n}\right\}$ be the ring of convergent power series at the origin in $\mathbb{C}^{n}$. Equisingular or topological classification of plane curve singularities is well-understood by ([Za1],[Za2]). In this paper, we only consider an analytic family of plane curves with isolated singularity defined as follows:

$f_{t}=f(y, z, t)=z^{n}+a_{1} z^{n-1}+\cdots+a_{n}$ for sufficiently small $t$ where the $a_{i}=$ $a_{i}(y, t) \in \mathbb{C}\{y, t\}, a_{i}(0, t)=0$ and $f(y, z, t)$ is square-free for each $t$.

O. Zariski gave a discriminant criterion for any analytic family of plane curve singularities of the above type to be equivalent, which is as follows:

Theorem([ZA2], Theorem 7, P.529). Consider an analytic family of plane curve singularities $C^{t}: f_{t}=f(y, z, t)=z^{n}+a_{1} z^{n-1}+\cdots+a_{n}$ for sufficiently small $t$ where the $a_{i}=a_{i}(y, t) \in \mathbb{C}\{y, t\}, a_{i}(0, t)=0$ and $f(y, z, t)$ is square-free for each $t$.

(a) A sufficient condition that $C^{0}$ and $C^{t}$ be equivalent is that the z-discriminant $\Delta(y, t)$ of $f(z, y, t)$ be of the form $\varepsilon(y, t) y^{N}$ where $\varepsilon(y, t)$ is a unit in $\mathbb{C}\{y, t\}$ and $N$ is a positive integer.

(b) If the line $y=0$ is not a tangent of $C^{0}$, then the above condition on $\Delta(y, t)$ is also necessary for the equivalence of $C^{t}$ and $C^{0}$.

The aim in this paper is to generalize this discriminant criterion, and it is very interesting to prove a generalized criterion, without using the proof of Zariski's discriminant criterion. In preparation for the generalization of Zariski's discriminant criterion, first of all, we need to prove the following:

THEOREM 3.2. Let $g=g(y, z)$ be a Weierstrass polynomial in $z$ at the origin of the form $z^{n}+b_{1} z^{n-1}+\cdots+b_{n}$ where $b_{i}$ are nonunits in $\mathbb{C}\{y\}$ for $1 \leq i \leq n$ and $g$ is square-free. Let the $z$-discriminant of $g$ be $\varepsilon(y) y^{N(g)}$ where $\varepsilon(y)$ is a unit in $\mathbb{C}\{y\}$ and $N(g)$ is a positive integer. Then we prove that $N(g)=\mu(g)+n-1$ where $\mu(g)$ is the Milnor number of the plane curve $\{g=0\}$ with an isolated singularity at the origin.

If $n$ is the multiplicity of $g(y, z)$ at the origin, then it was known by Theorem $2.8([\mathrm{Te} 1$, Proposition 1.2, p.317] $)$ that $N(g)=\mu(g)+n-1$.

* Received July 19, 2005; accepted for publication March 2, 2006.

$\dagger$ Department of Mathematics, Seoul National University, Seoul 151-742, Korea (chkang@math. snu.ac.kr). 
For the proof of Theorem 3.2, it is enough to use Theorem 2.8([Te1]), Theorem 2.7 ([Le-Ra $]$ ), which says that the invariance of Milnor's number implies the invariance of the topological type, and Theorem 2.5([Mi]) for the computation formula of the Milnor number, and the classical topological classification theorems for plane curve singularities, and Theorem 2.10([Ka]) for an equivalence of irreducible parametrization.

As an application of Theorem 3.2, replacing the condition that the line $y=0$ is not tangent to the plane curve $C^{0}$ by the condition that the regular order of $f(y, z, t)$ in $z$ at the origin in terms of Weierstrass polynomials is independent of $t$, we generalize Zariski's discriminant criterion as follows: The proof just follows from Theorem 3.2 and Theorem 2.7([Le-Ra]), again.

THEOREM 3.3. Consider an analytic family of plane curve singularities $C^{t}: f_{t}=$ $f(y, z, t)=z^{n}+a_{1} z^{n-1}+\cdots+a_{n}$ for sufficiently small $t$ where the $a_{i}=a_{i}(y, t) \in$ $\mathbb{C}\{y, t\}, a_{i}(0, t)=0$ and $f(y, z, t)$ is square-free for each $t$. Then $f_{t}$ is equisingular to $f_{0}$ if and only if the $z$-discriminant of $f_{t}$ is $y^{N}$ up to a unit factor in $\mathbb{C}\{y, t\}$ where $N$ is some positive integer not depending on $t$.

As another application of Theorem 3.2, a necessary condition of local irreducibility of plane curves with singularities can be easily found as follows:

THEOREM 4.1. Assume that $g=g(y, z)=z^{n}+b_{1} z^{n-1}+\cdots+b_{n}$ is a Weierstrass polynomial in $z$ where the $b_{i}$ are nonunits in $\mathbb{C}\{y\}$ and $g$ is square-free. Let the $z$ discriminant of $g$ be $y^{N(g)}$ up to a unit in $\mathbb{C}\{y\}$ where $N(g)$ is a positive integer. If $g$ is irreducible in $\mathbb{C}\{y, z\}$, then we get

$$
N(g) \not \equiv 0(\bmod n) .
$$

In other words, if $N(g) \equiv 0(\bmod n)$, then $g$ is reducible in $\mathbb{C}\{y, z\}$.

2. Known Preliminaries. Let ${ }_{n} \mathcal{O}$ or $\mathbb{C}\left\{z_{1}, \ldots, z_{n}\right\}$ be the ring of convergent power series at the origin in $\mathbb{C}^{n}$.

Definition 2.1. Let $V=\left\{z \in \mathbb{C}^{n+1}: f(z)=0\right\}$ and $W=\left\{z \in \mathbb{C}^{n+1}: g(z)=0\right\}$ be germs of complex analytic hypersurfaces with isolated singularity at the origin.

$f$ and $g$ are said to have the same topological type of singularity at the origin if there is a germ at the origin of homeomorphisms $\phi:\left(U_{1}, 0\right) \rightarrow\left(U_{2}, 0\right)$ such that $\phi(V)=W$ and $\phi(0)=0$ where $U_{1}$ and $U_{2}$ are open subsets in $\mathbb{C}^{n+1}$.

Lemma 2.2 (Hensel's Lemma). Let $f(y, z)=a_{0} z^{n}+a_{1} y^{\ell_{1}} z^{n-1}+\cdots+a_{n} y^{\ell_{n}}$ be irreducible in $\mathbb{C}\{y, z\}$ where each $a_{i}$ is a unit in $\mathbb{C}\{y, z\}$, if exists, and the $\ell_{i}$ are positive integers. Let $m$ be the multiplicity of $f$ at the origin. Then, $m=n$ or $\ell_{n}$. If $n=\ell_{i}+n-i$ for some $i$, then $n=\ell_{i}+n-i$ for all $i=1, \ldots, n$, and so $f$ can be written as follows: $f=f_{n}(y, z)+$ terms of degree $>n$, where $f_{n}$ is a homogeneous polynomials of degree $n$ with $f_{n}=(a y+b z)^{n}$ for some $a, b \in \mathbb{C}$.

Theorem 2.3([BR], [Bu], [ZA1]). As far as arbitrary Puiseux expansion of irreducible plane curve singularities is concerned, any two irreducible plane curve singularities have the same topological types if and only if they have the same type of the standard Puiseux expansion(or the same Puiseux pairs). In more detail, let $f(y, z)$ be irreducible in ${ }_{2} \mathrm{O}$ with an isolated singularity at the origin in $\mathbb{C}^{2}$. Then the standard 
Puiseux expansion topologically equisingular to the curve defined by $f$ at the origin can be described by $y=t^{n}$ and $z=t^{\alpha_{1}}+\cdots+t^{\alpha_{p}}$ where $n<\alpha_{1}<\cdots<\alpha_{p}$ and $n>\operatorname{gcd}\left(n, \alpha_{1}\right)>\cdots>\operatorname{gcd}\left(n, \alpha_{1}, \ldots, \alpha_{p}\right)=1$. If for a given $f$ there is another homeomorphic standard Puiseux expansion defined by $y=t^{m}$ and $z=t^{\beta_{1}}+\cdots+t^{\beta_{q}}$ where $m<\beta_{1}<\cdots<\beta_{q}$ and $m>\operatorname{gcd}\left(m, \beta_{1}\right)>\cdots>\operatorname{gcd}\left(m, \beta_{1}, \cdots \beta_{q}\right)=1$, then $n=m, p=q$ and $\alpha_{i}=\beta_{i}$ for $1 \leq i \leq p$.

THEOREM 2.4([LEJ], [ZA3]). Let $f(y, z)$ be in ${ }_{2} \mathcal{O}$ with an isolated singularity at the origin in $\mathbb{C}^{2}$. Then the topological type of the plane curve singularity defined by $f$ is determined by the topological type of every irreducible component of $f$ at $O$ and all the pairs of intersection multiplicity of these two components.

THEOREM 2.5([Mi]).

(1) Let $C$ be an irreducible curve parametrized by the Puiseux expansion

$$
C:=\left\{\begin{array}{l}
y=t^{n} \\
z=\lambda_{1} t^{a_{1}}+\lambda_{2} t^{a_{2}}+\lambda_{3} t^{a_{3}}+\cdots,
\end{array}\right.
$$

where

(1a) the exponents $a_{j}$ are positive integers with greatest common divisor one and with $2 \leq n<a_{1}<a_{2}<a_{3}<\cdots$, and the coefficients $\lambda_{j}$ are nonzero complex numbers,

(1b) let $d_{1}=\operatorname{gcd}\left(n, a_{1}\right), d_{2}=\operatorname{gcd}\left(n, a_{1}, a_{2}\right), \ldots, d_{j}=\operatorname{gcd}\left(n, a_{1}, a_{2}, \ldots, a_{j}\right)$ for each $j$, and then $d_{r}=1$ for sufficiently large $r$ such that $n=d_{0} \geq d_{1} \geq d_{2} \geq \cdots \geq$ $d_{r}=1$.

Then, the Milnor number $\mu(C)=2 \delta=\sum_{j \geq 1}\left(a_{j}-1\right)\left(d_{j-1}-d_{j}\right)$ with $d_{0}=n$.

Corollary 2.5.1([Mi]). First, let $C_{1}$ be the curve parametrized by the Puiseux expansion

$$
C_{1}:=\left\{\begin{array}{l}
y=t^{n} \\
z=t^{\alpha_{1}}+t^{\alpha_{2}}+\cdots+t^{\alpha_{r}},
\end{array}\right.
$$

where

(1a) $2 \leq n<\alpha_{1}<\alpha_{2}<\cdots<\alpha_{r}$,

(1b) $n \geq \operatorname{gcd}\left(n, \alpha_{1}\right)>\operatorname{gcd}\left(n, \alpha_{1}, \alpha_{2}\right)>\cdots>\operatorname{gcd}\left(n, \alpha_{1}, \alpha_{2}, \ldots, \alpha_{r}\right)=1$, and $n$ may be a divisor of $\alpha_{1}$,

(1c) write $d_{1}=\operatorname{gcd}\left(n, \alpha_{1}\right), d_{2}=\operatorname{gcd}\left(n, \alpha_{1}, \alpha_{2}\right), \ldots, d_{r-1}=\operatorname{gcd}\left(n, \alpha_{1}, \ldots, \alpha_{r-1}\right)$, $d_{r}=\operatorname{gcd}\left(n, \alpha_{1}, \ldots, \alpha_{r}\right)=1$.

In particular, if $n>\operatorname{gcd}\left(n, \alpha_{1}\right)$, note that the above parametrization is called the standard Puiseux expansion for the curve $C_{1}$.

As a conclusion, the Milnor number $\mu\left(C_{1}\right)=\sum_{j=1}^{r}\left(\alpha_{j}-1\right)\left(d_{j-1}-d_{j}\right)$ with $d_{0}=n$.

THEOREM 2.6([MI]). Let $f(y, z)$ be in $\mathbb{C}\{y, z\}$ with an isolated singularity at the origin in $\mathbb{C}^{2}$. Let $f(y, z)$ be a Weierstrass polynomial in $z$ at the origin of the form $z^{n}+a_{1} z^{n-1}+\cdots+a_{n}$ where $a_{i}$ are nonunits in $\mathbb{C}\{y\}$ for $1 \leq i \leq n$. If $f$ is reducible in $\mathbb{C}\{y, z\}$, then $f$ can be written as $f=f_{1} \cdots f_{h}$ where the $f_{i}$ are distinct irreducible Weierstrass polynomials in $z$ at the origin such that the $f_{i}$ are regular in 
z. By Milnor's formula ([Mi], Theorem 10.5, p. 85),

$$
\mu(f)=\mu\left(f_{1}\right)+\cdots+\mu\left(f_{h}\right)+2 \sum_{i<j} I\left(f_{i}, f_{j}\right)-h+1,
$$

where $I\left(f_{i}, f_{j}\right)$ is the intersection number of two distinct plane curves $\left\{f_{i}=0\right\}$ and $\left\{f_{j}=0\right\}$, and $h$ is the number of irreducible branches of $f$ at the origin.

THEOREM 2.7([LE-RA $])$. Let $F(t, z)$ be a polynomial in $z=\left(z_{0}, \ldots, z_{n}\right)$ with coefficients which are smooth complex valued functions of $t \in I=[0,1]$ such that $F(t, 0)=0$ and such that for each $t \in I$, the polynomials $\left(\partial F / \partial z_{i}\right)(t, z)$ in $z$ have an isolated zero at 0 . Assume moreover that the integer

$$
\mu_{t}=\operatorname{dim}_{\mathbb{C}} \mathbb{C}_{z} /\left(\frac{\partial f}{\partial z_{0}}(t, z), \ldots, \frac{\partial f}{\partial z_{n}}(t, z)\right)
$$

is independent of $t$. Then, the monodromy fibrations of the singularities of $F(0, z)=0$ and $F(1, z)=0$ at 0 are of the same fiber homotopy. If further $n \neq 2$, there fiberations are even differentiably isomorphic and the topological types of the singularities are the same.

Theorem 2.8 ([Te1, Proposition 1.2, P.317] or [Te2, Proposition, P.609]). Let $(X, 0) \subset\left(\mathbb{C}^{n+1}, 0\right)$ be a germ of hypersurface with an isolated singularity, and let $p:\left(\mathbb{C}^{n+1}, 0\right) \rightarrow(\mathbb{C}, 0)$ be a projection such that the fiber $\left(X_{0}, 0\right)$ of $\pi=p\lceil X$ again has an isolated singularity. Then, the multiplicity of the discriminant $D_{\pi}$ of $\pi$, denoted by $\Delta$, satisfies the following equality:

$$
\Delta=\mu^{(n+1)}(X, 0)+\mu^{(n)}\left(X_{0}, 0\right) .
$$

Note that the assumptions imply that the branch locus for a discriminant $D_{\pi}$ of $\pi$ is $\{0\}, i$. e., if we take a coordinate $z_{0}$ in $(\mathbb{C}, 0)$, then an equation for $D_{\pi}$ is $z_{0}{ }^{\Delta}=0$ with the following diagram:

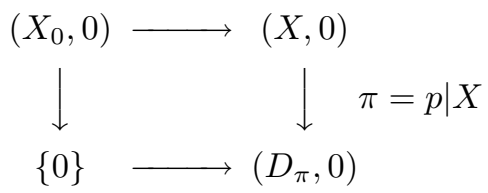

REMARK. $\mu^{(n+1)}(X, 0)=\mu(X, 0)$ and $\mu^{(1)}(X, 0)=m(X, 0)-1$ where $\mu(X, 0)$ is the Milnor number of $(X, 0)$ and $m(X, 0)$ is the multiplicity of $X$ at 0 .

Corollary 2.8.1 ([Te1, Corollary 1.5, P.320] or [Te2, Proposition, P.613]). Let $\left(X_{0}, 0\right) \subset\left(\mathbb{C}^{n+1}, 0\right)$ be a germ of hypersurace with an isolated singularity, which is defined by an equation $f\left(z_{0}, \ldots, z_{n}\right)=0$ in $\mathbb{C}\left\{z_{0}, \ldots, z_{n}\right\}$. Then, the multiplicity in $\mathcal{O}_{X_{0}, 0}$ of the jacobian ideal $j^{\prime}\left(=j(f) \cdot \mathcal{O}_{X_{0}, 0}\right)$ generated by the images of $\left(\frac{\partial f}{\partial z_{i}}\right) 0 \leq i \leq n$, is $\mu^{(n+1)}\left(X_{0}, 0\right)+\mu^{(n)}\left(X_{0}, 0\right)$. 
LEMma 2.9 (The REARRANGEMENT OF AN IRREDUCIBle PARAMETRIZATION, $[\mathrm{KA}])$.

Assumption Let the curve $V$ defined by $f(y, z) \in \mathbb{C}\{y, z\}$ have an irreducible parametrization as follows:

$$
y=t^{n} \text { and } z=c_{1} t^{k_{1}}+c_{2} t^{k_{2}}+\cdots
$$

where the $c_{i}$ are nonzero complex numbers and $1 \leq n, 1 \leq k_{1}<k_{2}<\cdots$, and $n \geq \operatorname{gcd}\left(n, k_{1}\right) \geq \operatorname{gcd}\left(n, k_{1}, k_{2}\right) \geq \cdots \geq \operatorname{gcd}\left(n, k_{1}, k_{2}, \ldots\right)=1$. To get a desired rearrangement of $y=t^{n}$ and $z=\sum_{i=1}^{\infty} c_{i} t^{k_{i}}$ in the conclusion of this lemma, first we can define a finite sequence $\left\{\alpha_{1}, \alpha_{2}, \ldots, \alpha_{r+1}\right\}$ from the sequence $\left\{k_{i}: i=1,2, \ldots\right\}$ consisting of the exponents $k_{i}$ in (2.9.1) as follows:

(1) Let $\alpha_{1}=k_{1}$, and then note that $n \geq \operatorname{gcd}\left(n, \alpha_{1}\right)$. That is, either $n=$ $\operatorname{gcd}\left(n, \alpha_{1}\right)$ or $n>\operatorname{gcd}\left(n, \alpha_{1}\right)$.

(2) Let $\alpha_{2}$ be the smallest positive integer among the exponents $k_{i}$ such that $n \geq \operatorname{gcd}\left(n, \alpha_{1}\right)>\operatorname{gcd}\left(n, \alpha_{1}, k_{i}\right)$.

(3) Let $\alpha_{3}$ be the smallest positive integer among the exponents $k_{i}$ such that $n \geq \operatorname{gcd}\left(n, \alpha_{1}\right)>\operatorname{gcd}\left(n, \alpha_{1}, \alpha_{2}\right)>\operatorname{gcd}\left(n, \alpha_{1}, \alpha_{2}, k_{i}\right)$.

$(\mathrm{r}+1)$ Let $\alpha_{r+1}$ be the smallest positive integer among the exponents $k_{i}$ such that $n \geq \operatorname{gcd}\left(n, \alpha_{1}\right)>\operatorname{gcd}\left(n, \alpha_{1}, \alpha_{2}\right)>\cdots>\operatorname{gcd}\left(n, \alpha_{1}, \alpha_{2}, \ldots, \alpha_{r}\right)>\operatorname{gcd}\left(n, \alpha_{1}, \alpha_{2}, \ldots\right.$, $\left.\alpha_{r}, k_{i}\right)=1$.

Let $d$ and $k$ be arbitrary positive integers. For brevity of notation, if $k$ is divisible by $d$, then we write $d \mid k$. Otherwise, we write $d \nmid k$.

Now, let $d_{i}=\operatorname{gcd}\left(n, \alpha_{1}, \ldots, \alpha_{i}\right)$ for $1 \leq i \leq r+1$, and then $n \geq d_{1}>d_{2}>\cdots>$ $d_{r+1}$. Note that $d_{i} \mid\left(\alpha_{i}-\alpha_{1}\right), d_{i} \backslash\left(\alpha_{i+1}-\alpha_{1}\right)$, and $d_{i+1} \mid d_{i}$.

Conclusion The given irreducible parametrization of $V$ can be rearranged in $t$ as follows:

$$
\begin{aligned}
y= & t^{n} \\
z= & c_{1} t^{\alpha_{1}}\left\{\left(1+c_{11} t^{d_{1}}+c_{12} t^{2 d_{1}}+\cdots+c_{1 p_{1}} t^{p_{1} d_{1}}\right)\right. \\
& +t^{\alpha_{2}-\alpha_{1}}\left(c_{20}+c_{21} t^{d_{2}}+c_{22} t^{2 d_{2}}+\cdots+c_{2 p_{2}} t^{p_{2} d_{2}}\right) \\
& +\ldots \ldots \\
& +t^{\alpha_{r}-\alpha_{1}}\left(c_{r 0}+c_{r 1} t^{d_{r}}+c_{r 2} t^{2 d_{r}}+\cdots+c_{r p_{r}} t^{p_{r} d_{r}}\right) \\
& \left.+t^{\alpha_{r+1}-\alpha_{1}}\left(c_{r+1,0}+\sum_{k+1}^{\infty} c_{r+1, k} t^{k}\right)\right\}
\end{aligned}
$$

satisfying the properties (i), (ii) and (iii).

(i) $c_{10}=1, c_{20}, c_{30}, \ldots, c_{r+1,0}$ are all nonzero complex numbers.

(ii) $p_{1}, p_{2}, \ldots, p_{r}$ are nonnegative integers such that

$$
\begin{aligned}
\alpha_{1}+p_{1} d_{1} & <\alpha_{2}<\alpha_{1}+\left(p_{1}+1\right) d_{1}, \\
\alpha_{2}+p_{2} d_{2} & <\alpha_{3}<\alpha_{2}+\left(p_{2}+1\right) d_{2}, \\
& \ldots \ldots \\
\alpha_{r-1}+p_{r-1} d_{r-1} & <\alpha_{r}<\alpha_{r-1}+\left(p_{r-1}+1\right) d_{r-1}, \\
\alpha_{r}+p_{r} d_{r} & <\alpha_{r+1}<\alpha_{r}+\left(p_{r}+1\right) d_{r} .
\end{aligned}
$$


(iii) Let $S$ be the set which consists of the remaining coefficients in $t$, that is,

$$
\begin{aligned}
S= & \left\{c_{11}, c_{12}, \ldots, c_{1, p_{1}}\right\} \cup\left\{c_{21}, c_{22}, \ldots, c_{2, p_{2}}\right\} \cup \cdots \\
& \cup\left\{c_{r 1}, c_{r 2}, \ldots, c_{r, p_{r}}\right\} \cup\left\{c_{r+1, k}: k=1,2, \ldots\right\} .
\end{aligned}
$$

Then, any element of $S$ is either zero or nonzero.

Note that $p_{i}$ may be zero for some $i, 1 \leq i \leq r$. In particular, if $p_{i}=0$ for $1 \leq i \leq r$, then note that $c_{i 1}, c_{i 2}, \ldots, c_{i, p_{i}}$ are all zero except for $c_{i 0}$.

TheOrem 2.10 (An EquivalenCE OF IRREDUCIBlE PARAMETRIZATION, [KA]). Assumption We may assume without loss of generality that the curve $V$ defined

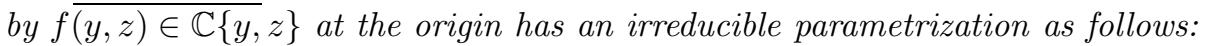

$$
\begin{aligned}
y= & t^{n}, \\
z= & c t^{\alpha_{1}}\left\{\left(1+D_{1}(t)\right)+t^{\alpha_{2}-\alpha_{1}}\left(c_{20}+D_{2}(t)\right)+\cdots\right. \\
& \left.+t^{\alpha_{r}-\alpha_{1}}\left(c_{r 0}+D_{r}(t)\right)+t^{\alpha_{r+1}-\alpha_{1}}\left(c_{r+1,0}+D_{r+1}(t)\right)\right\} \\
= & c t^{\alpha_{1}}(1+H(t)) \text { or } \\
y= & t^{n}, \\
z= & c t^{\alpha_{1}}\left\{1+D_{1}(t)\right\}+c t^{\alpha_{2}}\left\{c_{20}+D_{2}(t)\right\}+\cdots \\
& +c t^{\alpha_{r}}\left\{c_{r 0}+D_{r}(t)\right\}+c t^{\alpha_{r+1}}\left\{c_{r+1,0}+D_{r+1}(t)\right\}
\end{aligned}
$$

where

(i) $1 \leq n$ and $1 \leq \alpha_{1}<\alpha_{2}<\cdots<\alpha_{r+1}$,

(ii) $n \geq d_{1}>d_{2}>\cdots>d_{r+1}=1$ with $\operatorname{gcd}\left(n, \alpha_{1}, \alpha_{2}, \ldots, \alpha_{i}\right)=d_{i}$ for $1 \leq i \leq$ $r+1$,

(iii) $p_{1}, p_{2}, \ldots, p_{r}$ are nonnegative integers such that

$$
\begin{aligned}
& \alpha_{1}+p_{1} d_{1}<\alpha_{2}<\alpha_{1}+\left(p_{1}+1\right) d_{1}, \\
& \alpha_{2}+p_{2} d_{2}<\alpha_{3}<\alpha_{2}+\left(p_{2}+1\right) d_{2}, \\
& \quad \ldots \ldots \\
& \quad \ldots \ldots \alpha_{r-1}+p_{r-1} d_{r-1}<\alpha_{r}<\alpha_{r-1}+\left(p_{r-1}+1\right) d_{r-1}, \\
& \alpha_{r}+p_{r} d_{r}<\alpha_{r+1}<\alpha_{r}+\left(p_{r}+1\right) d_{r},
\end{aligned}
$$


(iv) let

$$
\begin{aligned}
D_{1}(t)= & \sum_{i=1}^{p_{1}} c_{1 i} t^{i d_{1}} \in \mathbb{C}[t], \\
D_{2}(t)= & \sum_{i=1}^{p_{2}} c_{2 i} t^{i d_{2}} \in \mathbb{C}[t], \\
\ldots & \ldots \\
D_{r}(t)= & \sum_{i=1}^{p_{r}} c_{r i} t^{i d_{r}} \in \mathbb{C}[t] \\
D_{r+1}(t)= & \sum_{k=1}^{\infty} c_{r+1, k} t^{k} \in \mathbb{C}\{t\} \\
1+H_{(}(t)= & 1+D_{1}(t)+t^{\alpha_{2}-\alpha_{1}}\left(c_{20}+D_{2}(t)\right)+\cdots \\
& +t^{\alpha_{r}-\alpha_{1}}\left(c_{r 0}+D_{r}(t)\right)+t^{\alpha_{r+1}-\alpha_{1}}\left(c_{r+1,0}+D_{r+1}(t)\right),
\end{aligned}
$$

(v) $c, c_{10}=1, c_{20}, c_{30}, \ldots, c_{r+1,0}$ are all nonzero complex numbers.

Conclusion We have the followings: Observe that (I) of two statements (I) and (II) below may be omitted, in order to simplify the statements for Conclusion, if necessary.

(I) In preparation for the construction of an equivalent irreducible parametrization of $V$, let $s$ be the new parameter defined by

$$
s(t)=c^{\frac{1}{\alpha_{1}}} t(1+H(t))^{\frac{1}{\alpha_{1}}}
$$

where

(i) $c^{\frac{1}{\alpha_{1}}}$ is a complex root such that $\omega^{\alpha_{1}}=c$,

(ii) $s=s(t)$ is a conformal mapping of $t$ at the origin,

(iii) $z=s^{\alpha_{1}}$.

Then $t=c^{-\frac{1}{\alpha_{1}}} s(1+H(t))^{-\frac{1}{\alpha_{1}}}$, as $t=\phi(s) \in \mathbb{C}\{s\}$, can be written as follows: Note that $y=(\phi(s))^{n}$.

$$
\begin{aligned}
t= & \phi(s) \\
= & c^{-\frac{1}{\alpha_{1}}} s\left\{1+Q_{1}(s)+s^{\alpha_{2}-\alpha_{1}}\left(B_{20}+Q_{2}(s)\right)\right. \\
& \left.+\cdots+s^{\alpha_{r}-\alpha_{1}}\left(B_{r 0}+Q_{r}(s)\right)+s^{\alpha_{r+1}-\alpha_{1}}\left(B_{r+1,0}+Q_{r+1}(s)\right)\right\},
\end{aligned}
$$


where

$$
\begin{aligned}
B_{20}= & \frac{c_{20}}{-\alpha_{1}}\left(c^{-\frac{1}{\alpha_{1}}}\right)^{\alpha_{2}-\alpha_{1}}, B_{30}=\frac{c_{30}}{-\alpha_{1}}\left(c^{-\frac{1}{\alpha_{1}}}\right)^{\alpha_{3}-\alpha_{1}}, \ldots, \\
& B_{r+1,0}=\frac{c_{r+1,0}}{-\alpha_{1}}\left(c^{-\frac{1}{\alpha_{1}}}\right)^{\alpha_{r+1}-\alpha_{1}}, \\
Q_{1}(s)= & B_{11} s^{d_{1}}+B_{12} s^{2 d_{1}}+\cdots+B_{1, p_{1}} s^{p_{1} d_{1}} \in \mathbb{C}[s], \\
Q_{2}(s)= & B_{21} s^{d_{2}}+B_{22} s^{2 d_{2}}+\cdots+B_{2, p_{2}} s^{p_{2} d_{2}} \in \mathbb{C}[s], \\
& \ldots \cdots \\
Q_{r}(s)= & B_{r 1} s^{d_{r}}+B_{r 2} s^{2 d_{r}}+\cdots+B_{r, p_{r}} s^{p_{r} d_{r}} \in \mathbb{C}[s], \\
Q_{r+1}(s)= & \sum_{k=1}^{\infty} B_{r+1, k} s^{k} \in \mathbb{C}\{s\}
\end{aligned}
$$

such that all the $B_{i j}$ are complex numbers and that in particular the $B_{i 0}$ are nonzero for $2 \leq i \leq r+1$. Note that $Q_{i}(0)=0$ for $1 \leq i \leq r+1$.

(II) The equivalent parametrization with the new parameter $s$ for $V$ can be analytically written in the following form:

$$
\begin{aligned}
z= & s^{\alpha_{1}}, \\
y= & c^{-\frac{n}{\alpha_{1}}} s^{n}\left\{1+Q_{1}^{*}(s)+s^{\alpha_{2}-\alpha_{1}}\left(b_{20}+Q_{2}^{*}(s)\right)\right. \\
& \left.+s^{\alpha_{3}-\alpha_{1}}\left(b_{30}+Q_{3}^{*}(s)\right)+\cdots+s^{\alpha_{r+1}-\alpha_{1}}\left(b_{r+1,0}+Q_{r+1}^{*}(s)\right)\right\},
\end{aligned}
$$

where

$$
\begin{aligned}
b_{20}= & \frac{n}{-\alpha_{1}} c_{20} c^{\frac{1}{-\alpha_{1}}\left(\alpha_{2}-\alpha_{1}\right)}, b_{30}=\frac{n}{-\alpha_{1}} c_{30} c^{\frac{1}{-\alpha_{1}}\left(\alpha_{3}-\alpha_{1}\right)}, \ldots, \\
& b_{r+1,0}=\frac{n}{-\alpha_{1}} c_{r+1,0} c^{\frac{1}{-\alpha_{1}}\left(\alpha_{r+1}-\alpha_{1}\right)}, \\
Q_{1}^{*}(s)= & b_{11} s^{d_{1}}+b_{12} s^{2 d_{1}}+\cdots+b_{1, p_{1}} s^{p_{1} d_{1}} \in \mathbb{C}[s], \\
Q_{2}^{*}(s)= & b_{21} s^{d_{2}}+b_{22} s^{2 d_{2}}+\cdots+b_{2, p_{2}} s^{p_{2} d_{2}} \in \mathbb{C}[s], \\
& \ldots \cdots \\
Q_{r}^{*}(s)= & b_{r 1} s^{d_{r}}+b_{r 2} s^{2 d_{r}}+\cdots+b_{r, p_{r}} s^{p_{r} d_{r}} \in \mathbb{C}[s], \\
Q_{r+1}^{*}(s)= & \sum_{k=1}^{\infty} b_{r+1, k} s^{k} \in \mathbb{C}\{s\}
\end{aligned}
$$

such that all the $b_{i j}$ are complex numbers and that in particular the $b_{i 0}$ are nonzero for $2 \leq i \leq r+1$. Note that $Q_{i}^{*}(0)=0$ for all $i=2,3, \ldots, r+1$.

Remark: Observe by (2.10.5) and (2.10.7) that

$\left(2.10 .7^{*}\right) \quad b_{20}=n B_{20}, b_{30}=n B_{30}, \ldots, b_{r+1,0}=n B_{r+1,0}$.

TheOREm 2.11([KA]). Suppose that the curve $V$ defined by $f(y, z) \in \mathbb{C}\{y, z\}$ at the origin satisfies the same assumptions and notations as in Theorem 2.10. First, let $C_{1}$ be the curve parametrized by the Puiseux expansion

$$
C_{1}:=\left\{\begin{array}{l}
y=t^{n} \\
z=t^{\alpha_{1}}+t^{\alpha_{2}}+\cdots+t^{\alpha_{r+1}},
\end{array}\right.
$$


where

(1a) $2 \leq n$ and $2 \leq \alpha_{1}<\alpha_{2}<\cdots<\alpha_{r+1}$,

(1b) $n \geq \operatorname{gcd}\left(n, \alpha_{1}\right)>\operatorname{gcd}\left(n, \alpha_{1}, \alpha_{2}\right)>\cdots>\operatorname{gcd}\left(n, \alpha_{1}, \alpha_{2}, \ldots, \alpha_{r+1}\right)=1$, and $n$ may be a divisor of $\alpha_{1}$,

(1c) write $d_{1}=\operatorname{gcd}\left(n, \alpha_{1}\right), d_{2}=\operatorname{gcd}\left(n, \alpha_{1}, \alpha_{2}\right), \ldots, d_{r}=\operatorname{gcd}\left(n, \alpha_{1}, \ldots, \alpha_{r}\right)$, $d_{r+1}=\operatorname{gcd}\left(n, \alpha_{1}, \ldots, \alpha_{r+1}\right)=1$.

Next, let $\mathrm{C}_{2}$ be the curve parametrized by the Puiseux expansion

$$
C_{2}:=\left\{\begin{array}{l}
y=t^{n}+t^{n+\alpha_{2}-\alpha_{1}}+t^{n+\alpha_{3}-\alpha_{1}}+\cdots+t^{n+\alpha_{r+1}-\alpha_{1}} \\
z=t^{\alpha_{1}} .
\end{array}\right.
$$

As a conclusion, $V(f), C_{1}$ and $C_{2}$ have the same topological type of singularity at the origin, satisfying the following property:

(i) If $n<\alpha_{1}$ and $n>\operatorname{gcd}\left(n, \alpha_{1}\right)$, then $C_{1}$ is the standard Puiseux expansion for the curve $V$.

(ii) If $n<\alpha_{1}$ and $n=\operatorname{gcd}\left(n, \alpha_{1}\right)$, then the parametrization defined by $y=t^{n}$ and $z=t^{\alpha_{2}}+\cdots+t^{\alpha_{r+1}}$ is the standard Puiseux expansion for the curve $V$.

(iii) If $n>\alpha_{1}$ and $\alpha_{1}>\operatorname{gcd}\left(n, \alpha_{1}\right)$, then $C_{2}$ is the standard Puiseux expansion for the curve $V$.

(iv) If $n>\alpha_{1}$ and $\alpha_{1}=\operatorname{gcd}\left(n, \alpha_{1}\right)$, then the parametrization defined by $y=$ $t^{n+\alpha_{2}-\alpha_{1}}+t^{n+\alpha_{3}-\alpha_{1}}+\cdots+t^{n+\alpha_{r+1}-\alpha_{1}}$ and $z=t^{\alpha_{1}}$ is the standard Puiseux expansion for the curve $V$.

Thus, the standard Puiseux expansion which is topologically equivalent to the Puiseux expansion of the curve $V(f)$ is uniquely determined.

The proof of Theorem 2.11 just follows from Theorem 2.3 and Theorem 2.10.

3. A discriminant criterion for an analytic family of an equivalence of plane curve singularities. Let ${ }_{n} \mathcal{O}$ or $\mathbb{C}\left\{z_{1}, \ldots, z_{n}\right\}$ be the ring of convergent power series at the origin in $\mathbb{C}^{n}$.

Definition 3.0. Let $\mathbb{C}\{y\}[z]$ be the polynomial ring in $z$ with coefficients in $\mathbb{C}\{y\}$ where $\mathbb{C}\{y\}$ is the ring of convergent power series centered at the origin. $f \in \mathbb{C}\{y\}[z]$ is said to be a Weierstrass polynomial of degree $n>0$ in $z$ if $f=z^{n}+\sum_{i=1}^{n} b_{i} z^{n-i}$ where for $1 \leq i \leq n$, the $b_{i}$ are nonunits in $\mathbb{C}\{y\}$. If $n$ is also the multiplicity of $f$ at the origin in $\mathbb{C}^{2}$, then it is said that $f$ is a Weierstrass polynomial of multiplicity $n$ in $z$.

REMARK 3.0.1. Let $f \in \mathbb{C}\{y\}[z]$ be a Weierstrass polynomial of degree $n>0$ in $z$. Observe that irreducibility in $\mathbb{C}\{y\}[z]$ is the same as irreducibility in $\mathbb{C}\{y, z\}$. If $f$ is reducible in $\mathbb{C}\{y, z\}$, then $f=f_{1} \cdots f_{k}$ where the $f_{i}$ are Weierstrass polynomials in $z$ and irreducible in $\mathbb{C}\{y, z\}$. Let $f_{i}=z^{n_{i}}+b_{1} z^{n_{i}-1}+\cdots+b_{n_{i}}$ for $1 \leq i \leq k$, where the $b_{i}$ are nonunits in $\mathbb{C}\{y\}$. Then, $n_{1}+n_{2}+\cdots+n_{k}=n$.

As an application of Theorem 2.8([Te2]), we have the following proposition:

Proposition 3.1. Let $f$ be a Weierstrass polynomial of multiplicity $n$ in $z$ which has the form $f=f(y, z)=z^{n}+a_{1} z^{n-1}+\cdots+a_{n}$ where the $a_{i}=a_{i}(y)$ are holomorphic near $y=0$ and $a_{i}(0)=0$ for $i=1, \cdots, n$. Let the line $\{z=0\}$ be tangent to the plane curve $\{f=0\}$ at the origin in $\mathbb{C}^{2}$. Assume that the discriminant of $f$ with respect to $z$ is not identically zero. Let the $z$-discriminant of $f$ be $y^{N(f)}$ up to a unit 
in $\mathbb{C}\{y\}$ where $\mathbb{C}\{y\}$ is the ring of convergent power series centered at the origin. Then $N(f)=\mu(f)+n-1$ where $\mu(f)$ is the Milnor number of the plane curve $\{f=0\}$ at the origin.

Proof of Proposition 3.1. In order to apply Theorem 2.8 to this proposition, let us look at the projection $p:\left(\mathbb{C}^{2}, 0\right) \rightarrow(\mathbb{C}, 0)$ defined by $(y, z) \mapsto y$. For convenience of proof, we may assume that $(X, 0)$ is a germ of reduced plane curve in $\left(\mathbb{C}^{2}, 0\right)$ where $X=\{(y, z): f(y, z)=0\}$. Note that the multiplicity of the plane curve $X$ at 0 is equal to the degree $n$ of a Weierstrass polynomial $f$ in $z$ at the origin. Then, $\pi=p \uparrow$ $X:(X, 0) \rightarrow(\mathbb{C}, 0)$ implies that the branch locus for a discriminant $D_{\pi}$ of $\pi$ is $\{0\}$. Now, let us consider a line $H=\{y=0\}$ in $\left(\mathbb{C}^{2}, 0\right)$ parallel to the projection $\pi$. Follow the same notations as in the proof of Theorem 2.8. Since $H$ is transversal to $(X, 0)$ by Hensel's lemma, then $\Delta=\mu^{(2)}(X, 0)+\mu^{(1)}(X \cap H, 0)=\mu^{(2)}(X, 0)+\mu^{(1)}(X, 0)=$ $\mu^{(2)}(X, 0)+m(X, 0)-1$ by Proposition 2.8 and Corollary 2.8.1 where $\mu^{(2)}(X, 0)=\mu(f)$ and $m(X, 0)$ is the multiplicity of $X$ at 0 for notation. Note by construction that $\Delta=\operatorname{dim}_{\mathbb{C}} \frac{\mathbb{C}\{y, z\}}{\left(f, f_{z}\right)}$ is equal to an integer $N(f)$ and $\mu^{(1)}(X \cap H, 0)=\operatorname{dim}_{\mathbb{C}} \frac{\mathbb{C}\{z\}}{f_{z}(0, z)}$ is equal to an integer $n-1$ and $m(X, 0)$ is equal to an integer $n$. Thus, the proof is done.

First we extend the above proposition, just replace mult $(f)$ by the regular order of $f$ in $z$. We start to define the regular order of $f$ in $z$. If $f$ is a Weierstrass polynomial of the form $f=z^{n}+a_{1} z^{n-1}+\cdots+a_{n}$ where the $a_{i}=a_{i}(y)$ are nonunits in $\mathbb{C}\{y\}$, then $f$ is said to be regular of order $n$ in $z$ at the origin. Observe that regular order is just degree of $f$ relative to $z$, which may not be equal to the multiplicity of $f$.

The main aim in this paper is to prove the following theorem, which generalize the above proposition.

THEOREM 3.2. Let $\mathbb{C}\{y\}$ be the ring of convergent power series centered at the origin. Let $f$ be a Weierstrass polynomial such that $f=z^{n}+a_{1} z^{n-1}+\cdots+a_{n}$ is regular of order $n$ in $z$ at the origin where the $a_{i}$ are nonunits in $\mathbb{C}\{y\}$ and square-free. Let the $z$-discriminant of $f$ be $y^{N}$ up to a unit in $\mathbb{C}\{y\}$. Then $N=\mu(f)+n-1$ where $\mu(f)$ is the Milnor number of the plane curve $\{f=0\}$ at the origin.

In preparation for the proof of Theorem 3.2, first we consider the case when $f$ of Theorem 3.2 is irreducible in $\mathbb{C}\{y, z\}$.

Proposition 3.2.1. Assume that $f=z^{n}+a_{1} z^{n-1}+\cdots+a_{n}$ is regular of order $n$ in $z$ at the origin where the $a_{i}$ are nonunits in $\mathbb{C}\{y\}$ and square-free. Assume that $f$ is irreducible in $\mathbb{C}\{y\}[z]$. Let the z-discriminant $R_{f, f_{z}}$ of $f$ be $y^{N(f)}$ up to a unit in $\mathbb{C}\{y\}$. Then $N(f)=\mu(f)+n-1$ where $\mu(f)$ is the Milnor number of the plane curve $\{f=0\}$ at the origin.

Proof of Proposition 3.2.1. Rewrite $f$ in the form:

$$
f=z^{n}+b_{1} y^{\ell_{1}} z^{n-1}+\cdots+b_{i} y^{\ell_{i}} z^{n-i}+\cdots+b_{n} y^{\ell_{n}}
$$

where $b_{i}$ are units in $\mathbb{C}\{y\}$ and $\ell_{i}$ are positive integers for $1 \leq i \leq n$. If the line $\{z=0\}$ is tangent to the plane curve $\{f=0\}$, there is nothing to prove by Proposition 3.1.

For the remaining of the proof, we may assume that the line $\{z=0\}$ is not tangent to the plane curve $\{f=0\}$, that is, $\ell_{n} \leq n$ by Hensel' Lemma.

Put

$$
g=z^{n}+b_{1} y^{\ell_{1}+1} z^{n-1}+\cdots+b_{i} y^{\ell_{i}+i} z^{n-i}+\cdots+b_{n} y^{\ell_{n}+n} .
$$


Since the line $\{z=0\}$ is tangent to the plane curve $\{g=0\}$, it is clear by Proposition 3.1 that $N(g)=\mu(g)+n-1$ where the $z$-discriminant $R_{g, g_{z}}$ of $g$ is defined to be $y^{N(g)}$ up to a unit in $\mathbb{C}\{y\}$, noting that $\mu(g)$ is the Milnor number of the plane curve $\{g=0\}$ at the origin. We use quadratic transformations or blow-ups ([L], Chap 1). Blowing up $\{g=0\}$ at $(0,0)$, we can get one and only one proper transform $\{f=0\}$ of the plane curve $\{g=0\}$. Observe that $g$ is also square-free.

Then, we claim the following:

claim[1] $\quad N(f)=N(g)-n(n-1)$.

claim[2] $\quad \mu(f)=\mu(g)-n(n-1)$.

If two claims are proved, then it is clear that $N(f)-\mu(f)=N(g)-\mu(g)=n-1$, and so the proof will be completely finished.

The proof of claim[1]: Observe that the proof of the claim[1] will be finished by Sublemma 1 and Sublemma 2.

Sublemma 1. Let

$$
\begin{aligned}
& F=A_{0} x_{k}^{n}+A_{1} x_{k}^{n-1}+\cdots+A_{n}, \\
& G=B_{0} x_{k}^{m}+B_{1} x_{k}^{m-1}+\cdots+B_{m},
\end{aligned}
$$

where $A_{i}$ and $B_{j}$ are homogeneous polynomials in $\mathbb{C}\left[x_{0}, x_{1}, \ldots, x_{k-1}\right]$, with degrees of $i$ and $j$, respectively. As a conclusion, if $R_{F, G} \in \mathbb{C}\left[x_{0}, x_{1}, \ldots, x_{k-1}\right]$ is the resultant of $F$ and $G$, then either $R_{F, G}$ is identically zero or $R_{F, G}$ is a homogeneous polynomial of degree of $n m$.

For the proof of Sublemma 1, see [BK, Proposition 8, p. 202].

An Example for Sublemma 1. In order to apply the result of Sublemma 1 to the proof of claim[1], consider the following example:

Let

$$
\begin{aligned}
& F=A_{0} z^{n}+A_{1} z^{n-1}+\cdots+A_{n}, \\
& G=n A_{0} z^{n-1}+(n-1) A_{1} z^{n-2}+\cdots+A_{n-1},
\end{aligned}
$$

where $G=F_{z}$, and $A_{i}$ are homogeneous polynomials in $\mathbb{C}\left[x_{0}, x_{1}, \ldots, x_{k-1}\right]$, with degrees of $i$, for $0 \leq i \leq n$. By Sublemma 1, it can be easily shown that if $R_{F, F_{z}}$ is viewed as a polynomial in $\mathbb{C}\left[A_{0}, A_{1}, \ldots, A_{n}\right]$, then $R_{F, F_{z}}$ has one and only one of the following:

(i) $R_{F, F_{z}}$ is identically zero.

(ii) Any nonzero monomial $A_{0}^{p_{0}} A_{1}^{p_{1}} A_{2}^{p_{2}} \cdots A_{n}^{p_{n}}$ of $R_{F, F_{z}}$ has $p_{1}+2 p_{2}+\cdots+n p_{n}=$ $n(n-1)$ where all the $p_{i}$ are nonnegative integers.

Sublemma 2. Rewrite $f$ in the form:

$$
f=z^{n}+b_{1} y^{\ell_{1}} z^{n-1}+\cdots+b_{i} y^{\ell_{i}} z^{n-i}+\cdots+b_{n} y^{\ell_{n}},
$$

where the $b_{i}$ are units in $\mathbb{C}\{y\}$ and the $\ell_{i}$ are positive integers for $1 \leq i \leq n$. Suppose the line $\{z=0\}$ is not tangent to the plane curve $\{f=0\}$ or $\ell_{n} \leq n$. Put

$$
g=z^{n}+b_{1} y^{\ell_{1}+1} z^{n-1}+\cdots+b_{i} y^{\ell_{i}+i} z^{n-i}+\cdots+b_{n} y^{\ell_{n}+n} .
$$


Since the line $\{z=0\}$ is tangent to the plane curve $\{g=0\}$. Let the $z$-discriminants of $f$ and $g$ be denoted by $R_{f, f_{z}}$ and $R_{g, g_{z}}$. As a conclusion, we have $R_{g, g_{z}}=y^{n(n-1)} R_{f, f_{z}}$

Proof of Sublemma 2. Put $b_{0}=1$. For any nonzero monomial $\prod_{i=0}^{n}\left(b_{i} y^{\ell_{i}}\right)^{p_{i}}$ in $R_{f, f_{z}}$ in the sense of an example for Sublemma 1 , then we can choose $\prod_{i=0}^{n}\left(b_{i} y^{\ell_{i}} y^{i}\right)^{p_{i}}$ in $R_{g, g_{z}}$, and conversely. Whenever $b_{i} y^{\ell_{i}} y^{i} z^{n-i}$ in $g$ is viewed as $c_{i} y^{i} z^{n-i}$, then it is clear by the above remark that $p_{1}+2 p_{2}+\cdots+n p_{n}=n(n-1)$, and so the proof of Sublemma 2 is done.

Thus, we can prove claim[1] by Sublemma 1 and Sublemma 2 .

The proof of claim[2]: The proof of claim[2] just follows from Sublemma 3.

Sublemma 3. Suppose that the same assumption as in Proposition 3.2 .1 holds. First, we construct the curve $C_{1}$ parametrized by the standard Puiseux expansion which is equisingular to $g(y, z)=0$ at the origin with the same tangent line $z=0$. Let $C_{1}$ be the curve parametrized by the standard Puiseux expansion

$$
C_{1}:=\left\{\begin{array}{l}
y=t^{n} \\
z=t^{\beta_{1}}+t^{\beta_{2}}+\cdots+t^{\beta_{r+1}},
\end{array}\right.
$$

where

(1a) $2 \leq n<\beta_{1}<\beta_{2}<\cdots<\beta_{r+1}$,

(1b) $n>\operatorname{gcd}\left(n, \beta_{1}\right)>\operatorname{gcd}\left(n, \beta_{1}, \beta_{2}\right)>\cdots>\operatorname{gcd}\left(n, \beta_{1}, \beta_{2}, \ldots, \beta_{r+1}\right)=1$,

(1c) write $d_{1}=\operatorname{gcd}\left(n, \beta_{1}\right), d_{2}=\operatorname{gcd}\left(n, \beta_{1}, \beta_{2}\right), \ldots, d_{r}=\operatorname{gcd}\left(n, \beta_{1}, \ldots, \beta_{r}\right)$, $d_{r+1}=\operatorname{gcd}\left(n, \beta_{1}, \ldots, \beta_{r+1}\right)=1$.

Let $\pi: M \rightarrow \mathbb{C}^{2}$ be a blow-up of $\mathbb{C}^{2}$ at $(0,0)$. Let $(v, u)$ and $\left(v^{\prime}, u^{\prime}\right)$ be the local coordinates for $M$ with $\pi(v, u)=(y, z)=(v, v u)$ and $\pi\left(v^{\prime}, u^{\prime}\right)=(y, z)=\left(v^{\prime} u^{\prime}, v^{\prime}\right)$ where $u^{\prime}=\frac{1}{u}$ and $v^{\prime}=v u$.

Let $C_{2}$ be the curve defined by the proper transform of $C_{1}$. Then, the curve $C_{2}$ can be parametrized by the Puiseux expansion

$$
C_{2}:=\left\{\begin{array}{l}
v=t^{n} \\
u=t^{\beta_{1}-n}+t^{\beta_{2}-n}+t^{\beta_{3}-n}+\cdots+t^{\beta_{r+1}-n} .
\end{array}\right.
$$

Since the curve $C_{2}$ and $f(y, z)=0$ at the origin have the same topological type of singularity, and also the line $\{z=0\}$ is not tangent to the plane curve $\{f=0\}$, then $\beta_{1}-n \leq n$.

As a conclusion, $\mu\left(C_{1}\right)-\mu\left(C_{2}\right)=n(n-1)$.

Proof of Sublemma 3. By Theorem 2.10 and Theorem 2.11, the curve $C_{2}$ can be topologically reparametrized by the Puiseux expansion

$$
C_{2}:=\left\{\begin{array}{l}
v=t^{n}+t^{n+\beta_{2}-\beta_{1}}+t^{n+\beta_{3}-\beta_{1}}+\cdots+t^{n+\beta_{r}-\beta_{1}} \\
u=t^{\beta_{1}-n}
\end{array}\right.
$$

By Corollary 2.5.1,

$$
\begin{aligned}
\mu\left(C_{1}\right)= & \left(\beta_{1}-1\right)\left(n-d_{1}\right)+\left(\beta_{2}-1\right)\left(d_{1}-d_{2}\right)+\left(\beta_{3}-1\right)\left(d_{2}-d_{3}\right) \\
& +\cdots+\left(\beta_{n}-1\right)\left(d_{n-1}-d_{n}\right), \\
\mu\left(C_{2}\right)= & (n-1)\left(\beta_{1}-n-d_{1}\right)+\left(n+\beta_{2}-\beta_{1}-1\right)\left(d_{1}-d_{2}\right) \\
& +\left(n+\beta_{3}-\beta_{1}-1\right)\left(d_{2}-d_{3}\right)+\cdots+\left(n+\beta_{n}-\beta_{1}-1\right)\left(d_{n-1}-d_{n}\right) .
\end{aligned}
$$


Compute $\mu\left(C_{1}\right)-\mu\left(C_{2}\right)$. Then,

$$
\begin{aligned}
\mu\left(C_{1}\right)-\mu\left(C_{2}\right)= & \left\{\left(\beta_{1}-1\right)\left(n-d_{1}\right)-(n-1)\left(\beta_{1}-n-d_{1}\right)\right\} \\
& +\left\{\left(\beta_{2}-1\right)\left(d_{1}-d_{2}\right)-\left(n+\beta_{2}-\beta_{1}-1\right)\left(d_{1}-d_{2}\right)\right\} \\
& +\left\{\left(\beta_{3}-1\right)\left(d_{2}-d_{3}\right)-\left(n+\beta_{3}-\beta_{1}-1\right)\left(d_{2}-d_{3}\right)\right\}+\cdots \\
& +\left\{\left(\beta_{n}-1\right)\left(d_{n-1}-d_{n}\right)-\left(n+\beta_{n}-\beta_{1}-1\right)\left(d_{n-1}-d_{n}\right)\right\} \\
= & \left\{\left(\beta_{1}-1\right)\left(n-d_{1}\right)-(n-1)\left(\beta_{1}-n-d_{1}\right)\right\} \\
& -\left\{\left(n-\beta_{1}\right)\left(d_{1}-d_{2}\right)+\left(n-\beta_{1}\right)\left(d_{2}-d_{3}\right)+\cdots\right. \\
& \\
= & \left.\left(\beta_{1}-1\right)\left(n-d_{1}\right)-(n-1)\left(\beta_{1}-n-d_{1}\right)-\left(n-d_{n-1}\right)\left(d_{1}-1\right)\right\} \\
= & \left(\beta_{1}-1\right)\left(n-d_{1}\right)-(n-1)\left(\beta_{1}-n\right)+(n-1) d_{1}-\left(n-\beta_{1}\right)\left(d_{1}-1\right) \\
= & n(n-1) .
\end{aligned}
$$

Thus, the proof of Sublemma 3 is done. So, we can prove claim[2].

Therefore, the proof of Proposition 3.2.1 is completely finished.

Proof of Theorem 3.2. Rewrite $f$ in the form:

$$
f=z^{n}+b_{1} y^{\ell_{1}} z^{n-1}+\cdots+b_{i} y^{\ell_{i}} z^{n-i}+\cdots+b_{n} y^{\ell_{n}},
$$

where $b_{i}$ are units in $\mathbb{C}\{y\}$ and $\ell_{i}$ are positive integers for $1 \leq i \leq n$. If the line $\{z=0\}$ is tangent to the plane curve $\{f=0\}$, there is nothing to prove by Proposition 3.1.

For the remaining of the proof, we may start to assume that the line $\{z=0\}$ is not tangent to the plane curve $\{f=0\}$ or $\ell_{n} \leq n$.

Put

$$
g=z^{n}+b_{1} y^{\ell_{1}+1} z^{n-1}+\cdots+b_{i} y^{\ell_{i}+i} z^{n-i}+\cdots+b_{n} y^{\ell_{n}+n} .
$$

But, note that the line $\{z=0\}$ is tangent to the plane curve $\{g=0\}$. We use quadratic transformations or blow-ups ([La], Chap 1). Blowing up $\{g=0\}$ at $(0,0)$, we get one and only one proper transform $\{f=0\}$ of the plane curve $\{g=0\}$. Observe that $g$ is also square-free. Let the $z$-discriminant of $g$ be $y^{N(g)}$ up to a unit factor in $\mathbb{C}\{y\}$ where $N(g)$ is some positive integer.

We claim the following:

(1) $N(g)=\mu(g)+n-1$ where $g$ is irreducible in $\mathbb{C}\{y, z\}$ and the line $\{z=0\}$ is tangent to the plane curve $\{g=0\}$ at the origin, which is trivial to prove.

(2) $N(f)=N(g)-n(n-1)$.

(3) $\mu(f)=\mu(g)-n(n-1)$.

If $f$ is irreducible in $\mathbb{C}\{y, z\}$, then it was already proved by Proposition 3.2.1.

Let the line $\{z=0\}$ be not tangent to the plane curve $\{f=0\}$ and $f$ be reducible in $\mathbb{C}\{y, z\}$. Then $f$ can be written as $f=f_{1} \cdots f_{h}$ where the $f_{i}$ are irreducible Weierstrass polynomials and regular in $z$. From the construction of $g$ in (3.2.9), $g$ can be written as $g=g_{1} \cdots g_{h}$ where the $g_{i}$ are irreducible Weierstrass polynomials and regular in $z$. Moreover, if $\left\{f_{i}=0\right\}$ is the corresponding proper transform of $\left\{g_{i}=0\right\}$ for each $i$, then note that the intersection number of $\left\{g_{i}=0\right\}$ and $\left\{g_{j}=0\right\}$ decreases by $\left(\operatorname{mult}\left(g_{i}\right)\right) \cdot\left(\operatorname{mult}\left(g_{j}\right)\right)$ for $i \neq j$, after one time blow-up. See [Fu, p.74] for the definition of the intersection number. Let $N\left(f_{i}\right)$ be the total order of the zero to the 
$z$-discriminant of $f_{i}$ at $y=0$, and $N\left(g_{i}\right)$, that of $g_{i}, 1 \leq i \leq h$. Let $\mu\left(f_{i}\right)$ be the Milnor number of $f_{i}$ and $\mu\left(g_{i}\right)$, that of $g_{i}$ for $i=1, \ldots, h$.

So, we get the following:

$$
\begin{aligned}
N\left(f_{i}\right) & =\mu\left(f_{i}\right)+n_{i}-1 & & \text { by Proposition } 3.2 .1, \\
N\left(g_{i}\right) & =\mu\left(g_{i}\right)+n_{i}-1 & & \text { by Proposition } 3.2 .1, \\
\mu\left(g_{i}\right)-\mu\left(f_{i}\right) & =n_{i}\left(n_{i}-1\right) & & \text { by Sublemma } 3,
\end{aligned}
$$

and $n_{1}+\cdots+n_{h}=n$ where the $n_{i}$ is the multiplicity of $g_{i}$ at the origin, and also the regular order of $f_{i}$ in $z$ at the origin. By Milnor's formula (Theorem 2.6),

$$
\mu(g)=\mu\left(g_{1}\right)+\cdots+\mu\left(g_{h}\right)+2 \sum_{i<j} I\left(g_{i}, g_{j}\right)-h+1,
$$

where $I\left(g_{i}, g_{j}\right)$ is the intersection number of two distinct plane curves $\left\{g_{i}=0\right\}$ and $\left\{g_{j}=0\right\}$, and $h$ is the number of irreducible branches of $g$ at the origin.

By (3.2.10) and (3.2.11), we get

$$
\begin{aligned}
\mu(g)= & \mu\left(f_{1}\right)+n_{1}\left(n_{1}-1\right)+\cdots+\mu\left(f_{h}\right)+n_{h}\left(n_{h}-1\right) \\
& +2 \sum_{i<j} I\left(f_{i}, f_{j}\right)+2 \sum_{i<j} n_{i} n_{j}-h+1 .
\end{aligned}
$$

Observe that

$$
\begin{aligned}
& n_{1}\left(n_{1}-1\right)+\cdots+n_{h}\left(n_{h}-1\right)+2 \sum_{i<j} n_{i} n_{j} \\
& =\left(n_{1}+\cdots+n_{h}\right)^{2}-\left(n_{1}+\cdots+n_{h}\right)=n(n-1) .
\end{aligned}
$$

By (3.2.12) and (3.2.13), we get that $\mu(g)=\mu(f)+n(n-1)$ using Milnor's formula for $\mu(f)=\mu\left(f_{1} \cdots f_{h}\right)$ in the sense of (3.2.11). Since $N(g)=N(f)+n(n-1)$ by the proof of claim[1] or Sublemma 2, then we have $N(f)-\mu(f)=N(g)-\mu(g)$, which must be equal to $n-1$. Thus, the proof of the theorem is completely finished.

THEOREM 3.3. Consider an analytic family of plane curve singularities $C^{t}: f_{t}=$ $f(y, z, t)=z^{n}+a_{1} z^{n-1}+\cdots+a_{n}$ for sufficiently small $t$ where the $a_{i}=a_{i}(y, t) \in$ $\mathbb{C}\{y, t\}, a_{i}(0, t)=0$ and $f(y, z, t)$ is square-free for each $t$. Then $f_{t}$ is equisingular to $f_{0}$ if and only if the $z$-discriminant of $f_{t}$ is $y^{N}$ up to a unit factor in $\mathbb{C}\{y, t\}$ where $N$ is some integer not depending on $t$. Ra]).

Proof of Theorem 3.3. It just follows from Theorem 3.2 and Theorem 2.7([Le-

\section{A necessary condition of local irreducibility of plane curves with singularities.}

THEOREM 4.1. Assume that $f=z^{n}+a_{1} z^{n-1}+\cdots+a_{n}$ is a Weierstrass polynomial in $z$ where the $a_{i}$ are nonunits in $\mathbb{C}\{y\}$ and $f$ is square-free. Let the $z$ discriminant of $f$ be $\varepsilon(y) y^{N(f)}$ where $N(f)$ is a positive integer and $\varepsilon(y)$ is a unit in $\mathbb{C}\{y\}$. If $f$ is irreducible in $\mathbb{C}\{y, z\}$, then we get

$$
N(f) \not \equiv 0(\bmod n) \text {. }
$$


Proof of Theorem 4.1. In order to compute the number $N$, it suffices to consider two cases, respectively:

Case (i): The line $\{z=0\}$ is tangent to the plane curve $\{f=0\}$.

Case (ii): The line $\{z=0\}$ is not tangent to the plane curve $\{f=0\}$.

Case (i): Let the line $\{z=0\}$ be tangent to the plane curve $\{f=0\}$. To compute the number $N(f)$, then we may assume by Theorem 2.3, Theorem 2.10 and Theorem 2.11 that $\{f=0\}$ has the same topological type near the origin as the curve defined by the Puiseux expansion, that is,

$$
\left\{\begin{array}{l}
y=t^{n} \\
z=t^{\alpha_{1}}+t^{\alpha_{2}}+\cdots+t^{\alpha_{r+1}},
\end{array}\right.
$$

where $2 \leq n<\alpha_{1}<\alpha_{2}<\cdots<\alpha_{r+1}$ and $d_{0}=n \geq d_{1}=\operatorname{gcd}\left(n, \alpha_{1}\right)>d_{2}=$ $\operatorname{gcd}\left(n, \alpha_{1}, \alpha_{2}\right)>\cdots>d_{r+1}=\operatorname{gcd}\left(n, \alpha_{1}, \cdots, \alpha_{r+1}\right)=1$. Note that $n$ may be a divisor of $\alpha_{1}$.

Now, since $f$ is irreducible in $\mathbb{C}\{y, z\}$ together with the same hypotheses as in Proposition 3.1, using Corollary 2.5.1, we compute $N(f)=\mu(f)+n-1$ as follows:

(a) Let $r+1=1$ or $r=0$. Then $N(f)=\mu(f)+n-1=\left(\alpha_{1}-1\right)(n-1)+$ $n-1=\left(\alpha_{1}-1\right) n+n-\alpha_{1}$. Since $d_{1}=\operatorname{gcd}\left(n, \alpha_{1}\right)=1$, there is nothing to prove for $N(f) \not \equiv 0(\bmod n)$.

(b) Let $r+1 \geq 2$. Then, we have

$$
\begin{aligned}
N(f)= & \mu(f)+n-1 \\
= & \left(\alpha_{1}-1\right)\left(d_{0}-d_{1}\right)+\left(\alpha_{2}-1\right)\left(d_{1}-d_{2}\right)+\cdots \\
& +\left(\alpha_{r+1}-1\right)\left(d_{r}-d_{r+1}\right)+n-1 \\
= & \text { some multiple of } d_{r}-\left(\alpha_{r+1}-1\right)+n-1 \\
= & \text { some multiple of } d_{r}+n-\alpha_{r+1} .
\end{aligned}
$$

Since $\alpha_{r+1}$ is relatively prime to $d_{r}=\operatorname{gcd}\left(n, \alpha_{1}, \cdots, \alpha_{r}\right)$, we get

$$
N(f) \not \equiv 0\left(\bmod \operatorname{gcd}\left(n, \alpha_{1}, \cdots, \alpha_{r}\right)\right) .
$$

So, $N(f) \not \equiv 0(\bmod n)$. Thus, the proof of Case (i) is done.

Case (ii): Let the line $\{z=0\}$ is not tangent to the plane curve $\{f=0\}$. Rewrite $f$ in the form

$$
f=z^{n}+b_{1} y^{\ell_{1}} z^{n-1}+\cdots+b_{i} y^{\ell_{i}} z^{n-i}+\cdots+b_{n} y^{\ell_{n}},
$$

where $b_{i}$ are units in $\mathbb{C}\{y\}$ and $\ell_{i}$ are positive integers for $1 \leq i \leq n$.

As in the proof of Theorem 3.2, put

$$
g=z^{n}+b_{1} y^{\ell_{1}+1} z^{n-1}+\cdots+b_{i} y^{\ell_{i}+i} z^{n-i}+\cdots+b_{n} y^{\ell_{n}+n} .
$$

Note that the line $\{z=0\}$ is tangent to the plane curve $\{g=0\}$. We use quadratic transformations or blow-ups ([La], Chap 1). Blowing up $\{g=0\}$ at $(0,0)$, we get one and only one proper transform $\{f=0\}$ of the plane curve $\{g=0\}$. Observe that $g$ is also square-free. Let the $z$-discriminant of $g$ be $y^{N(g)}$ up to a unit factor in $\mathbb{C}\{y\}$ where $N(g)$ is some positive integer. By Case $(\mathrm{i}), N(g) \not \equiv 0(\bmod n)$. Since 
$N(f)=N(g)-n(n-1)$ by Sublemma 2 of Proposition 3.2.1, there is nothing to prove. Thus, the proof of Case (ii) is done, and so the proof of the theorem is finished.

Corollary 4.2. Under the same hypotheses of Theorem 4.1, if $N(f) \equiv 0(\bmod n)$ then $f$ is reducible in $\mathbb{C}\{y, z\}$.

Now we give some examples:

(1) Let $f=\left(z^{3}+y^{4}\right)^{2}+y^{7} z^{2}$ or let $f$ be topologically and parametrically given by $y=t^{6}$ and $z=t^{8}+t^{13}$. Then $\mu(f)=40$ and the $z$-discriminant of $f$ is $y^{N(f)}$ up to a unit factor in $\mathbb{C}\{y\}$ with $N(f)=45$. Note that $f$ is irreducible in $\mathbb{C}\{y, z\}$ and $N(f) \not \equiv 0(\bmod 6)$.

(2) Let $f=\left(z^{2}+y^{7}\right)\left(z^{3}+y^{4}\right)$. Then the $z$-discriminant of $f$ is $y^{N(f)}$ up to a unit factor in $\mathbb{C}\{y\}$ with $N(f)=31$. But, $N(f) \not \equiv 0(\bmod 5)$.

\section{REFERENCES}

[Br] Brauner, K., Zur Geometrie der Funktionen Zweier Komplexen Vesänderlicken, Abh. Math. Sem. Hamburg, 6 (1928), pp. 1-54.

[Bri-Kn] Brieskorn, E. And Knorrer, H., Plane Algebraic Curves Vol.I, Vol.II, Birkhauser Verlag Basel, 1986.

[Bu] Burau, W., Kennzeichung der Schlauchknoten, Abh. Math. Sem. Hamburg, 9 (1932), pp. $125-133$.

[En-Ch] Enriques, F And Chisini, O., Lezioni sulla teoria geometrica delle equazioni e delle funzioni algebriche, 3 Vols., Bologna, 1915, 1918, 1924.

[Fu] Fulton, W., Algebraic curves, The Benjamin Publishing Company, Inc. 1969.

[Ha] Hartshorne, R., Algebraic Geometry, Springer-Verlag, N.Y. 1977.

[Ka] KANG, C., An equivalence for irreducible parametrization and its application to the direct proof of an equivalent of the Puiseux pairs and the multiplicity sequences for irreducible curves, Asian J. Math., 3 (2003), pp. 365-434.

[La] Laufer, H., Normal two-dimensional singularities, Annals of Math. Studies, No. 71, Princeton University Press, Princeton 1971.

[Le-Ra] Le Dung Trang et C.P. Ramanujan, The invariance of Milnor's number implies the invariance of the topological type, Amer. J. Math., 98, I (1976), pp. 67-78.

[Lej] Lejeune, M., Sur l' equivalence des singularité des courbes algebroides planes, Coefficients de Newton, Centre de Math. del 1'Ecole Polytechnique, 1969.

[Mi] Milnor, J., Singular points of complex hypersurfaces, Annals of Math. Studies, No. 61, Princeton University Press, 1968.

[Re] ReEve, J. E., A summary of results in the topological classification of plane algebroid singularities, Rendiconti Seminario Matematico Universita e Politecnico Torino, 14 (1955), pp. 159-187.

[Te 1] Teissier, B., Cycles evanescents, sections planes et conditions de Whitney, Asterisque, 7-8 (1973).

[Te 2] Teissier, B., Introduction to equisingularity problems, Proceedings of Symposia in Pure mathematics, 29 (1975), pp. 593-632.

[Za 1] ZaRiski, O., On the topology of algebroid singularities, Amer. J. Math., 54 (1932), pp. 433465.

[Za 2] ZARISKI, O., Studies in equisingularity I, Equivalent singularities of plane algebroid curves, Amer. J. Math., 87 (1965), pp. 507-536.

[Za 3] Zariski, O., Studies in equisingularity III, Amer. J. Math., 90 (1968), pp. 961-1023. 